\title{
Possession and Utilization Pattern of Cashless Payment Methods by Respondents
}

\author{
Manisha Ohlan and Ella Rani*
}

Department of Extension Education and Communication Management, I.C. College of Home Sciences, CCS Haryana Agricultural University, Hisar, Haryana, India

*Corresponding author

\section{A B S T R A C T}

\begin{tabular}{|l|}
\hline Ke y w o r d s \\
Cashless system, \\
Possession, \\
Utilization, \\
Bankcards \\
\hline Article Info \\
\hline $\begin{array}{l}\text { Accepted: } \\
\text { 24 May } 2019 \\
\text { Available Online: } \\
\text { 10 June } 2019\end{array}$ \\
\hline
\end{tabular}

\section{Keywords}

\section{Introduction}

Cash and cashless have become the buzzwords of the day, ever since Prime Minister Modi's surprise announcement on the evening of $8^{\text {th }}$ November, 2016. Overnight, all the Rs. 500 and Rs. 1,000 notes in the country became doomed for destruction. Thus, began their journey, next morning to the banks, petrol pumps, railway ticket counters and few other places where they would find temporary acceptance. And, as we recovered from the initial shock, even disbelief, and got used to manage with less cash, we slowly woke up to the realization that the withdrawal of cash is part of a bigger, grander agenda of the government to ultimately eradicate cash from the society and become a cashless one (Jacob, 2016).

Today, credit cards and on-line payment services have become increasingly in style in urban Asian nation, paper currency notes are still an important part of life. Money could also be outlined as any legal medium of exchange that is like a shot negotiable and freed from restrictions. Indians are the fourth-largest user of money in the world. As most of the individuals are engaged in tiny transactions and having less 
banking habits, it is the most convenient and straight forward variety of medium of exchange, free from inconvenience, etc.

A less-cash economy comes with its own advantages such as an increased utilization of e-payment which includes faster transactions through reduction in queue at the banking halls, elimination of bacterial spread through handling of notes and coins from one individual to another, increment in sales by saving 20 per cent, curbed armed robbery and cash related crime.

It is widely believed that the movement from cash to cashless economy has significant benefits. Moody's Analytics (2013) studied the impact of card usage on gross domestic product (GDP) of 51 countries and found that electronic card usage added USD 1.1 trillion in real dollars to private consumption and GDP from 2003 to 2008. Further, study found that 1 per cent increase in card transaction volume would increase consumption each year by 0.039 per cent and GDP growth by 0.024 per cent (Moody's Analytics, 2013). Present study was planned to explore possession and utilization of cashless payment methods by respondents.

\section{Materials and Methods}

The study was conducted in Hisar district of Haryana state selected purposively in both rural and urban areas. For rural respondents, from Hisar district two villages; Ludas and Shahpur were selected randomly. For urban respondents, two localities; Sector-14 and Sector-15 from Hisar city were selected randomly to assess constraints about cash less system of the respondents. A total of 200 respondents which comprised of 100 rural respondents ( 25 females, 25 males) from each village and 100 urban respondents (25females, 25 males) from each locality having at least higher secondary education were selected purposively. A well-structured interview schedule was used as a tool for possession and utilization of cash less payment methods as a tool for data collection.

\section{Results and Discussion}

\section{Possession of cashless payment methods by respondents}

Payment methods possessed in rural and urban area has been presented in Table 1. It can be seen from the Table that in rural area, majority of the respondents $(57 \%)$ possessed ATM, followed by debit cards (28\%), credit cards $(26 \%)$, internet banking (24\%), PayTm (6\%), cheque (9\%), National Electronic Funds Transfer and demand draft (6\% each), whereas equal number of respondents $(2 \%$ each) had Unstructured Supplementary Service Data, Immediate Payments Service and Real Time Gross Settlement System.

While, in urban area, 91 per cent of the respondents had possession of ATM, followed by credit card (72\%), cheque $(65 \%)$, debit card (50\%), Immediate Payments Service $(34 \%)$, demand draft $(30 \%)$, National Electronic Funds Transfer and internet banking (21\% each), BHIM app (18\%), Unstructured Supplementary Service Data, Real Time Gross Settlement System and PayTm (12\% each), Electronic Clearance Service (10\%) and MobiKwik (2\%) respectively.

In case of pooled sample, nearly $3 / 4^{\text {th }}$ of the respondents (74\%) had possession of ATM, followed by debit card (54\%), credit card (49\%), cheque (37\%), internet banking (22.5\%), Immediate Payments Service and demand draft (18\% each), BHIM app and PayTm (9\%), Unstructured Supplementary Service Data and Real Time Gross Settlement Service System (7\% each), Electronic Clearance Service (5\%) and MobiKwik (1\%). 
Table.1 Possession of cashless payment methods by respondents

\begin{tabular}{|c|c|c|c|c|}
\hline \multirow{2}{*}{$\begin{array}{l}\text { Sr. } \\
\text { No. }\end{array}$} & \multirow[t]{2}{*}{ Payment Methods } & Rural & Urban & Total \\
\hline & & $\begin{array}{c}(\%) \\
(n=100)\end{array}$ & $\begin{array}{c}(\%) \\
(n=100)\end{array}$ & $\begin{array}{c}\mathbf{f}(\%) \\
(\mathbf{N}=\mathbf{2 0 0})\end{array}$ \\
\hline 1. & \multicolumn{4}{|l|}{ Bankcards } \\
\hline i. & Credit card & 26.00 & 72.00 & $98(49.00)$ \\
\hline ii. & Debit card & 28.00 & 50.00 & $108(54.00)$ \\
\hline iii. & ATM card & 57.00 & 91.00 & $148(74.00)$ \\
\hline iv. & $\begin{array}{l}\text { Unstructured Supplementary Service Data } \\
\text { (USSD) }\end{array}$ & 02.00 & 12.00 & $14(07.00)$ \\
\hline v. & Immediate Payments Service (IMPS) & 02.00 & 34.00 & $36(18.00)$ \\
\hline vi. & Electronic Clearance Service (ECS) & 00.00 & 10.00 & $10(05.00$ \\
\hline vii. & National Electronic Fund Transfer (NEFT) & 06.00 & 21.00 & $27(13.50)$ \\
\hline viii. & Real Time Gross Settlement (RTGS) & 02.00 & 12.00 & $14(07.00)$ \\
\hline ix. & Cheque & 09.00 & 65.00 & $74(37.00)$ \\
\hline $\mathrm{x}$. & Demand Draft & 06.00 & 30.00 & $36(18.00)$ \\
\hline 2. & \multicolumn{4}{|l|}{ Mobile apps } \\
\hline i. & BHIM & 00.00 & 18.00 & $18(09.00)$ \\
\hline ii. & MobiKwik & 00.00 & 02.00 & $02(01.00)$ \\
\hline iii. & PayTm & 06.00 & 12.00 & $18(09.00)$ \\
\hline 3. & Internet banking & 24.00 & 21.00 & $45(22.50)$ \\
\hline
\end{tabular}

Figures in parentheses indicate percentage 
Table.2 Utilization of cashless payment methods by respondents

\begin{tabular}{|c|c|c|c|c|c|c|c|c|c|c|c|c|c|}
\hline \multirow{2}{*}{$\begin{array}{l}\text { Sr. } \\
\text { No. }\end{array}$} & \multirow{2}{*}{$\begin{array}{l}\text { Payment } \\
\text { Methods }\end{array}$} & \multicolumn{5}{|c|}{ Rural $(n=100)$} & \multicolumn{5}{|c|}{ Urban $(n=100)$} & \multicolumn{2}{|c|}{ Total $(\mathrm{N}=\mathbf{2 0 0})$} \\
\hline & & $\begin{array}{l}\text { Always } \\
\text { (3) }\end{array}$ & $\begin{array}{c}\text { Sometimes } \\
\text { (2) }\end{array}$ & $\begin{array}{l}\text { Rarely } \\
\text { (1) }\end{array}$ & $\begin{array}{l}\text { Weighted } \\
\text { Mean } \\
\text { Score }\end{array}$ & Rank & $\begin{array}{c}\text { Always } \\
\text { (3) }\end{array}$ & $\begin{array}{c}\text { Sometimes } \\
\text { (2) }\end{array}$ & $\begin{array}{l}\text { Rarely } \\
\text { (1) }\end{array}$ & $\begin{array}{l}\text { Weighted } \\
\text { Mean } \\
\text { Score }\end{array}$ & Rank & $\begin{array}{l}\text { Weighted } \\
\text { Mean } \\
\text { Score }\end{array}$ & Rank \\
\hline 1. & \multicolumn{13}{|l|}{ Bankcards } \\
\hline i. & Credit card & 04 & 20 & 02 & 0.54 & III & 14 & 44 & 14 & 1.44 & II & 0.990 & II \\
\hline ii. & Debit card & 05 & 21 & 02 & 0.59 & II & 10 & 15 & 25 & 0.85 & IV & 0.720 & III \\
\hline iii. & ATM card & 17 & 32 & 08 & 1.23 & I & 60 & 21 & 10 & 2.11 & I & 1.670 & I \\
\hline iv. & $\begin{array}{l}\text { Unstructured } \\
\text { Supplementary } \\
\text { Service } \\
\text { Data(USSD) }\end{array}$ & 00 & 02 & 00 & 0.04 & VIII & 04 & 04 & 4 & 0.24 & XII & 0.140 & $\mathrm{XI}$ \\
\hline v. & $\begin{array}{l}\text { Immediate } \\
\text { Payments Service } \\
\text { (IMPS) }\end{array}$ & 00 & 02 & 00 & 0.04 & VIII & 04 & 15 & 15 & 0.57 & VI & 0.305 & VII \\
\hline vi. & $\begin{array}{l}\text { Electronic } \\
\text { Clearance Service } \\
\text { (ECS) }\end{array}$ & 00 & 02 & 00 & 0.04 & VIII & 00 & 04 & 06 & 0.14 & XIII & 0.090 & XII \\
\hline vii. & $\begin{array}{l}\text { National } \\
\text { Electronic Fund } \\
\text { Transfer (NEFT) }\end{array}$ & 00 & 06 & 00 & 0.12 & VI & 04 & 14 & 03 & 0.43 & VII & 0.275 & VIII \\
\hline viii. & $\begin{array}{l}\text { Real Time Gross } \\
\text { Settlement } \\
\text { (RTGS) }\end{array}$ & 00 & 02 & 00 & 0.04 & VIII & 07 & 03 & 02 & 0.29 & $\mathrm{X}$ & 0.165 & $\mathrm{X}$ \\
\hline ix. & Cheque & 01 & 08 & 00 & 0.19 & $\mathrm{~V}$ & 10 & 30 & 25 & 1.15 & III & 0.670 & IV \\
\hline $\mathrm{x}$. & Demand Draft & 01 & 05 & 00 & 0.08 & VII & 10 & 16 & 04 & 0.66 & $\mathrm{~V}$ & 0.370 & VI \\
\hline 2. & \multicolumn{13}{|l|}{ Mobile apps } \\
\hline i. & BHIM & 00 & 00 & 00 & 0.00 & - & 03 & 12 & 03 & 0.36 & IX & 0.180 & IX \\
\hline ii. & MobiKwik & 00 & 00 & 00 & 0.00 & - & 00 & 2 & 00 & 0.04 & XIV & 0.020 & XIII \\
\hline iii. & PayTm & 00 & 06 & 00 & 0.12 & VI & 04 & 05 & 03 & 0.25 & $\mathrm{XI}$ & 0.370 & VI \\
\hline 3. & Internet banking & 08 & 12 & 04 & 0.53 & IV & 03 & 08 & 10 & 0.38 & VIII & 0.455 & $\mathrm{~V}$ \\
\hline
\end{tabular}


The results are supported by Khan (2010) indicated the significant dimensions of ATM service quality as convenience, efficient operation, security and privacy, reliability and responsiveness. It also reflected a positive and strong relationship between ATM services and customers' satisfaction.

\section{Utilization of cashless payment methods by respondents}

The data presented in Table 2 reveals that cashless payment methods utilized by respondents in rural and urban area. It was found that in rural area, ATM card was utilized by majority of the respondents which was given first rank, which was followed by debit card (II ${ }^{\text {nd }}$ rank), credit card (III ${ }^{\text {rd }}$ rank), internet banking (IV ${ }^{\text {th }}$ rank), cheque $\left(\mathrm{V}^{\text {th }}\right.$ rank), National Electronic Funds Transfer and PayTm (VI ${ }^{\text {th }}$ rank), demand draft (VII ${ }^{\text {th }}$ rank) and Immediate Payments Service, Electronic Clearance Service and Real Time Gross Settlement System (VIII ${ }^{\text {th }}$ rank).

Data in Table further shows that in urban area, ATM card was utilized by majority of the respondents which was given first rank, which was followed by credit card (II ${ }^{\text {nd }}$ rank), cheque (III ${ }^{\text {rd }}$ rank), debit card (IV ${ }^{\text {th }}$ rank), demand draft $\left(\mathrm{V}^{\text {th }}\right.$ rank), Immediate Payments Service (VI ${ }^{\text {th }}$ rank), National Electronic Funds Transfer (VII ${ }^{\text {th }}$ rank), internet banking (VIII ${ }^{\text {th }}$ rank), BHIM app (IX ${ }^{\text {th }}$ rank), Real Time Gross Settlement System ( $X^{\text {th }}$ rank), PayTm $\left(\mathrm{XI}^{\text {th }}\right.$ rank), Unstructured Supplementary Service Data (XII ${ }^{\text {th }}$ rank), Electronic Clearance Service (XIII ${ }^{\text {th }}$ rank) and MobiKwik (XIV ${ }^{\text {th }}$ rank).

In case of pooled sample score, first rank was given to ATM card, which was followed by credit card (II ${ }^{\text {nd }}$ rank), debit card (III ${ }^{\text {rd }}$ rank), cheque (IV ${ }^{\text {th }}$ rank), internet banking ( $\mathrm{V}^{\text {th }}$ rank), PayTm and demand draft $\left(\mathrm{VI}^{\mathrm{th}}\right.$ rank each), Immediate Payments Service (VII ${ }^{\text {th }}$ rank), National Electronic Funds Transfer (VIII $^{\text {th }}$ rank), BHIM (IX ${ }^{\text {th }}$ rank), Real Time Gross Settlement System ( $X^{\text {th }}$ rank), Unstructured Supplementary Service Data $\left(\mathrm{XI}^{\text {th }}\right.$ rank), Electronic Clearance Service (XII $^{\text {th }}$ rank) and MobiKwik (XIII ${ }^{\text {th }}$ rank).Similar results are reported in World Payment Report (2016) that debit and credit cards have been a preferred method of payment for numerous years. Debit cards accounted for the largest share of global noncash transactions in 2014 was the most rapid growth method of payment.

Thus, it can be concluded that majority of respondents had possession of ATM card, followed by debit and credit card and first rank was given to ATM card, followed by credit card and debit card as cashless payments method were utilized by the respondents.

\section{References}

Khan, A.M., 2010. An empirical study of ATM service quality and customer satisfaction in Pakistani bank. European Journal of Social Sciences, 2(8):104109.

Moody's Analytics. 2013. The Impact of Electronic Payments on Economic Growth. https://usa.visa.com/dam/VCOM/downl oad/corporate/media/moodys-economywhite-paper-feb-2013.pdf

\section{How to cite this article:}

Manisha Ohlan and Ella Rani. 2019. Possession and Utilization Pattern of Cashless Payment Methods by Respondents. Int.J.Curr.Microbiol.App.Sci. 8(06): 3036-3040.

doi: https://doi.org/10.20546/ijcmas.2019.806.362 